\title{
COMPACTNESS OF THE EMBEDDING OPERATORS FOR ROUGH DOMAINS
}

\author{
VLADIMIR GOL’DSHTEIN AND ALEXANDER G. RAMM
}

\begin{abstract}
New classes of non-smooth bounded domains $D$, for which the embedding operator from $H^{1}(D)$ into $L^{2}(D)$ is compact, are introduced. These classes include, in particular, the domains whose boundary locally are graphs of $C$ - functions, but also contain much larger classes of domains. Examples of non-smooth domains for which the above embedding is compact are given. Applications to scattering by rough obstacles are mentioned.
\end{abstract}

Mathematics subject classification (2000): 46E39, 30C60. domains.

Key words and phrases: Sobolev spaces, Embedding theorems, Quasiconformal mappings, Rough

\section{REFERENCES}

[1] Sh. Agmon, Lectures on Elliptic Boundary Value Problems. D.Van Nostrand Co. Princeton, Toronto, New York, London, 1965.

[2] R. Courant, D. Hilbert, Methoden der mathematischen Physik, vol 2, Springer Verlag, Berlin, 1937.

[3] W. Evans, D. HARRIS, Sobolev embeddings for generalized ridged domains, Proc. Lond. Math. Soc., 54, 3, (1987), 141-175.

[4] F. W. Gehring and J. Vaisala, Hausdorff dimension and quasiconformal mappings, J. London Math.Soc. (2),6, (1973), 504-521.

[5] V. M. GOL'DShTEIN, S. K. VODOP'JANOV, Prolongement des functions de classe $L(2,1)$ et applications quasiconformes, C. R. Ac. Sc., Paris, 290, 10, (1980), A453-456.

[6] V. M. Gol'DShTEIN, Yu. G. ReSheTNYAK, Quasiconformal Mappings and Sobolev Spaces, Kluwer Academic Publishers. Dordrecht, Boston, London. 1990.

[7] V. Gol'dshtein, L. GuROV, A. Romanov, Homeomorphisms that induce monomorphisms of Sobolev spaces, Isr. J. of Math., 91, (1995), 31-60.

[8] V. GOL'DSHTEIN AND L. GUROv, Applications of change of variable operators for exact embedding theorems, Integr. Equat. Oper. Th., 19, (1994), 1-24.

[9] L. E. FRAENKEL, On regularity on the boundary in the theory of Sobolev spaces, London Math. Soc., 39, (1979), 385-427.

[10] V. MAZ'YA, Sobolev Spaces, Springer Verlag, Berlin, 1985.

[11] S. PoborchiI, V. G. MaZ'Ya, Differentiable functions on bad domains, World Scient., Singapore, 1997.

[12] A. G. RAMM, A necessary and sufficient condition for compactness of embedding, Vestnik Leningradskogo Univ., ser. math., mech. and astron., 1, (1963), 150-151.

[13] A. G. RAMM, M. SAMMARTINO, Existence and uniqueness of the scattering solutions in the exterior of rough domains, in the book "Operator theory and applications", Amer. Math. Soc. and Fields Institute, Providence, 2000, pp. 457-472.

[14] A. G. RAMM AND A. RUIZ, Existence and uniqueness of the scattering solutions in non-smooth domains, J. Math. Anal. Appl., 201, (1996), 329-338.

[15] W. P. ZIEMER, Weakly Differentiable Functions, Springer Verlag, 1989 